\title{
The 9th advanced summer school on service-oriented computing, June 28-July 3, Hersonissos, Crete, Greece
}

\author{
Wolfgang Reisig 1
}

Published online: 19 December 2016

(C) Springer-Verlag Wien 2016

The 9th Advanced Summer School on Service-Oriented Computing (SummerSOC'15) continued a successful series of summer schools that started in 2007, regularly attracting world-class experts in Service-Oriented Computing to present state-of-the-art research during a week-long program organized in four thematic tracks: formal methods for SOC; computing in the clouds; elasticity in the cloud; and emerging topics. The advanced summer school is regularly attended by top researchers from academia and industry as well as by graduate students from programs with international acclaim, such as the Erasmus Mundus International Master in Service Engineering.

During the morning sessions at SummerSOC renowned researchers gave invited tutorials on subjects from the themes mentioned above. The afternoon sessions were dedicated to presentations of original research contributions in these areas: accepted contributions were submitted in advance and have been peer-reviewed. Four of these contributions have been distinguished and selected to be published as articles in this special issue.

Zimmermann is taking a decision-centric view on cloud migration striving towards architectural refactoring for the cloud. Vucojevic-Haupt et al. present technology to provision process-centric applications on demand in the cloud. Hirmer et al. introduce situation templates to support situation recognition. Steimle et al. discuss issues in realizing a health-data-management system in the cloud in a secure manner.

Wolfgang Reisig

reisig@informatik.hu-berlin.de

1 Berlin, Germany 